\title{
Review of Extensor Mechanism Injuries in the Dislocated Knee
}

\author{
Munachukwudi Okoye, BS ${ }^{1}$ Abigail Wissman, BS ${ }^{2}$ Robert D. Wissman, MD ${ }^{1}$ \\ ${ }^{1}$ Department of Radiology, University of Missouri, Columbia, Missouri \\ ${ }^{2}$ Ohio University Heritage College of Osteopathic Medicine, Athens, \\ Ohio \\ Address for correspondence Robert D. Wissman, MD, Department of \\ Radiology, University of Missouri, Columbia, MO 65211 \\ (e-mail: rwissman@health.missouri.edu).
}

J Knee Surg 2022;35:498-501.

\begin{abstract}
Keywords

- knee dislocation

- extensor mechanism

- magnetic resonance imaging

Knee dislocations, aka multiligamentous injuries, are uncommon but devastating injuries often following high-energy trauma. Tears of the major knee stabilizers are well documented; however, injuries of the knee extensor mechanism are less commonly reported. The extensor mechanism is comprised of the patella, patellar tendon, and quadriceps tendons. Magnetic resonance imaging (MRI) is the preferred imaging modality of internal derangements of the knee due to its excellent soft tissue contrast. In this article, we will discuss the normal imaging findings of the extensor mechanism and review abnormalities following knee dislocation.
\end{abstract}

Knee dislocations, referring to disruption of the tibiofemoral articulation, are uncommon but devastating injuries. They most commonly occur following high-energy trauma; however, low energy causes, such as sports, have been implicated as well. ${ }^{1,2}$ Dislocations often occur in younger individuals and may reduce spontaneously prior to clinical or radiological evaluation. The prevalence of tibiofemoral dislocations is therefore likely underestimated owing to their propensity to reduce spontaneously. Tibiofemoral dislocations frequently result in damage to the major stabilizers of the knee, thereby increasing the risk of injury to the popliteal artery and peroneal nerve. ${ }^{3,4}$

Knee dislocations can be categorized by the position of the tibia in respect to the femur. The most common presentation is anterior dislocation usually due to a hyperextension injury, followed by posterior dislocations occurring from an anterior directed force to the flexed knee. Less commonly, the knee can dislocate medially, laterally, or rotationally. ${ }^{5}$ Multiple ligaments may be injured most commonly involving the anterior and posterior cruciate ligaments with medial and lateral ligament tears less commonly identified. These injuries can be classified by the Schenk system which is the preferred method of grading knee dislocations - Table 1.

Extensor mechanism injuries are also possible; however, they are infrequently discussed in the literature. The purpose of this article is to review the normal imaging findings of the

received

July 14, 2021

accepted after revision

December 23, 2021

published online

February 28, 2022

extensor mechanism, as well as abnormalities, following dislocation of the knee joint.

\section{Normal Imaging}

Conventional radiography is often the initial imaging modality for the assessment of the acutely injured knee. Following multiligamentous injuries, its main utility is to exclude fractures and assess joint alignment. Abnormal positioning of the patella may be a secondary indicator of extensor mechanism rupture ${ }^{6,7}$ (-Fig. 1). Magnetic resonance imaging (MRI), with its superior soft tissue contrast remains the modality of choice for the assessment of internal derangements of the knee joint.

All ligaments and tendons are predominantly low in signal on all MRI pulse sequences. A laminated appearance, however, is often present in the distal insertion of the normal quadriceps tendon as a result of the convergence of individual tendon fibers. The rectus femoris is located most anteriorly, the vastus medialis and lateralis comprise the intermediate layer, and the vastus intermedius is the deepest portion of the tendon. The vastus medialis can be subdivided into the vastus medialis oblique (VMO) and vastus medialis longus. The normal VMO muscle should be nonedematous and flush against the femur. The patellar tendon also is

(c) 2022. Thieme. All rights reserved. Thieme Medical Publishers, Inc., 333 Seventh Avenue, 18th Floor, New York, NY 10001, USA
DOI https://doi.org/ $10.1055 / \mathrm{s}-0042-1743224$. ISSN 1538-8506. 
Table 1 Schenck's classification

\begin{tabular}{|l|l|}
\hline Classification & Description \\
\hline KD-I & $\mathrm{ACL}$ or $\mathrm{PCL}+$ collateral \\
\hline KD-II & $\mathrm{ACL}+\mathrm{PCL}$ \\
\hline KD-III M & $\mathrm{ACL}+\mathrm{PCL}+\mathrm{MCL}$ \\
\hline KD-III L & $\mathrm{ACL}+\mathrm{PCL}+\mathrm{LCL}$ \\
\hline KD-IV & $\mathrm{ACL}+\mathrm{PCL}+\mathrm{MCL}+\mathrm{LCL}$ \\
\hline KD-V & Fracture dislocation \\
\hline
\end{tabular}

Abbreviations: $\mathrm{ACL}$, anterior cruciate ligament; $\mathrm{PCL}$, posterior cruciate ligament; KD, knee dislocation; $M C L$, medial cruciate ligament; LCL, lateral cruciate ligament.

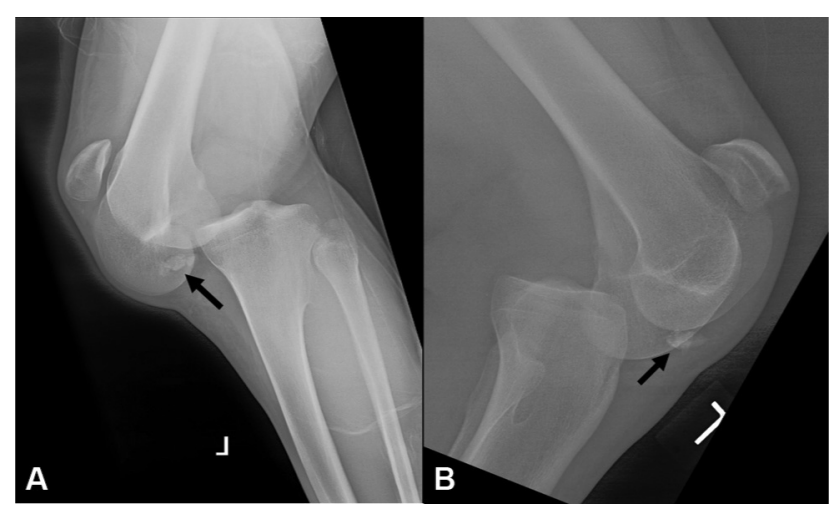

Fig. 1 Frontal (A) and lateral (B) radiographs of a 24-year-old male with a posterior knee dislocation following a fall. Note the avulsion fracture of the inferior pole of the patella resulting in patella alta (curved arrow) suggesting patellar tendon rupture. The patient was taken directly to the operating room for patellar fracture fixation and patellar tendon repair.

predominantly low in signal on all pulse sequences except for two small triangular regions of the deep tendon proximally, at the origin with the patella, and distally at its tibial insertion $^{7,8}$ (-Fig. 2).
The patella plays an important role in the protection of the distal femoral articular cartilage and increases the mechanical leverage during extension by elevating the extensor tendons from the axis of rotation of the knee. ${ }^{8}$ The patella should be centrally located in the trochlear groove on axial MRIs. The patellofemoral joint should be congruent without abnormal tilting of the patella. On sagittal images, the patella can be easily identified between the junction of the quadriceps and patellar tendons.

The medial patellofemoral ligament (MPFL) is an hourglass-shaped ligament that acts as the major stabilizer of the patella. Its origin is a triangular space between the adductor tubercle, medial femoral epicondyle, and gastrocnemius tubercle, and its insertion is the superomedial aspect of the patella. ${ }^{9}$ At MRI, the normal MPFL is a bilaminated, low signal structure intimately associated with the VMO muscle, and is best visualized on axial imaging (-Fig. $\mathbf{3}$ ).

\section{Patella Injuries}

\section{Patella Fractures}

Fractures of the patella are uncommon accounting for less than $2 \%$ of all skeletal injuries. ${ }^{10}$ The most common mechanism is direct impact to the knee such as in a fall. The patella is a sesamoid bone embedded in the quadriceps tendon. This subcutaneous position of the patella increases the potential of an open patella fracture or injury from a direct blow. ${ }^{11}$ The diagnosis is uncomplicated as patients will often present with swelling, pain, and reduced active extension in the affected knee. ${ }^{12}$ In most cases, radiographs are sufficient, however, nondisplaced fractures may require MRI for proper diagnosis.

\section{Patellar Instability}

Knee dislocations may produce MRI finding similar to isolated patellar dislocation. This includes VMO elevation and edema, MPFL tears, and abnormal patellar alignment. ${ }^{13}$ MPFL tears are common and are present in the majority of knee dislocators. Similar to isolated patellar dislocations, the most common site of injury is the femoral origin of the
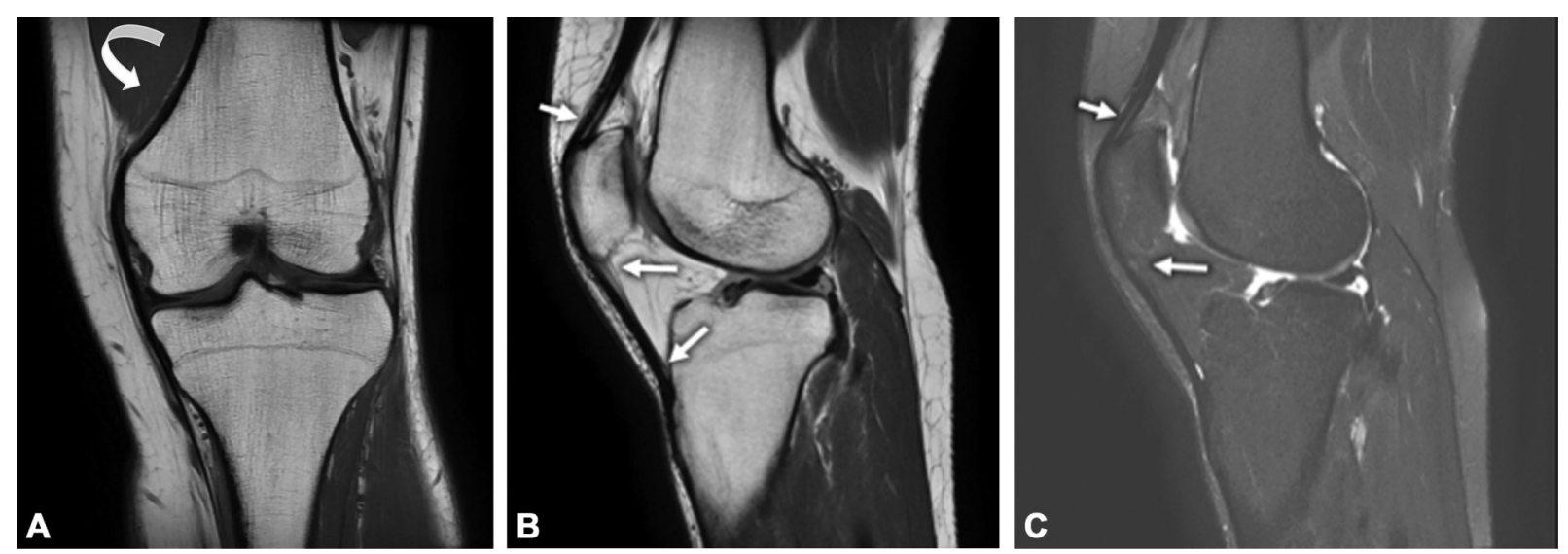

Fig. 2 Normal knee structures in a 24-year-old female. Coronal T1-weighted image (A) demonstrates the VMO muscle flush against the distal femur (arrow). Sagittal T1-weighted (B) and T2-weighted (C) images demonstrate the normal laminar appearance of the quadriceps tendon (curved arrow) and normal triangular regions of increased signal in the proximal and distal patellar tendons (straight arrows). VMO, vastus medialis oblique. 


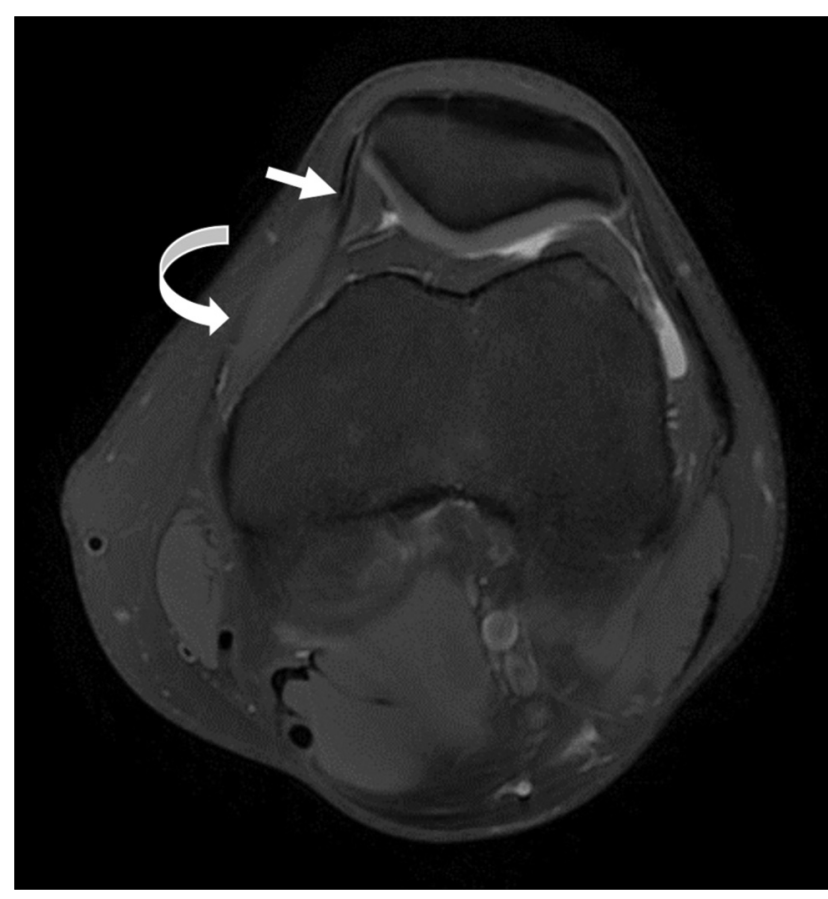

Fig. 3 Normal knee structures in a 24-year-old female. Axial T2weighted image demonstrate the patella centered in the trochlea. Note the normal VMO muscle (curved arrow) and the normal bilaminar appearance of the MPFL (straight arrow). MPFL, medial patellofemoral ligament; VMO, vastus medialis oblique.

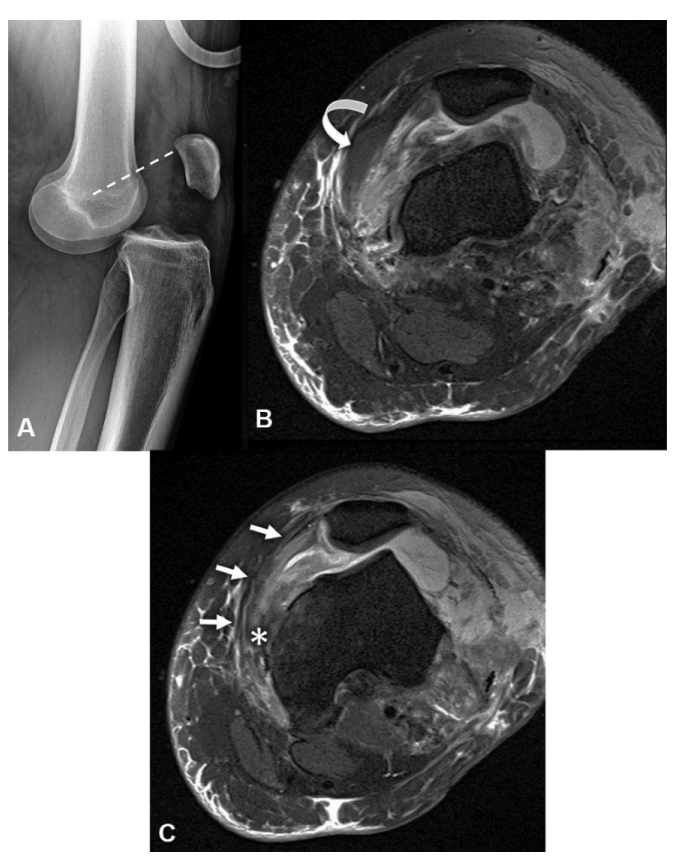

Fig. 4 A 34-year-old male with posterior knee dislocation. Lateral knee radiograph demonstrates elongation of the MPFL dashed line (A). Axial T2-weighted images (B, C) demonstrate elevation of the VMO (curved arrow) and elongation of the MPFL (arrows) with a hematoma at the femoral origin of the ligament $\left({ }^{*}\right)$. Note the lateral subluxation of the patella. MPFL, medial patellofemoral ligament; VMO, vastus medialis oblique.

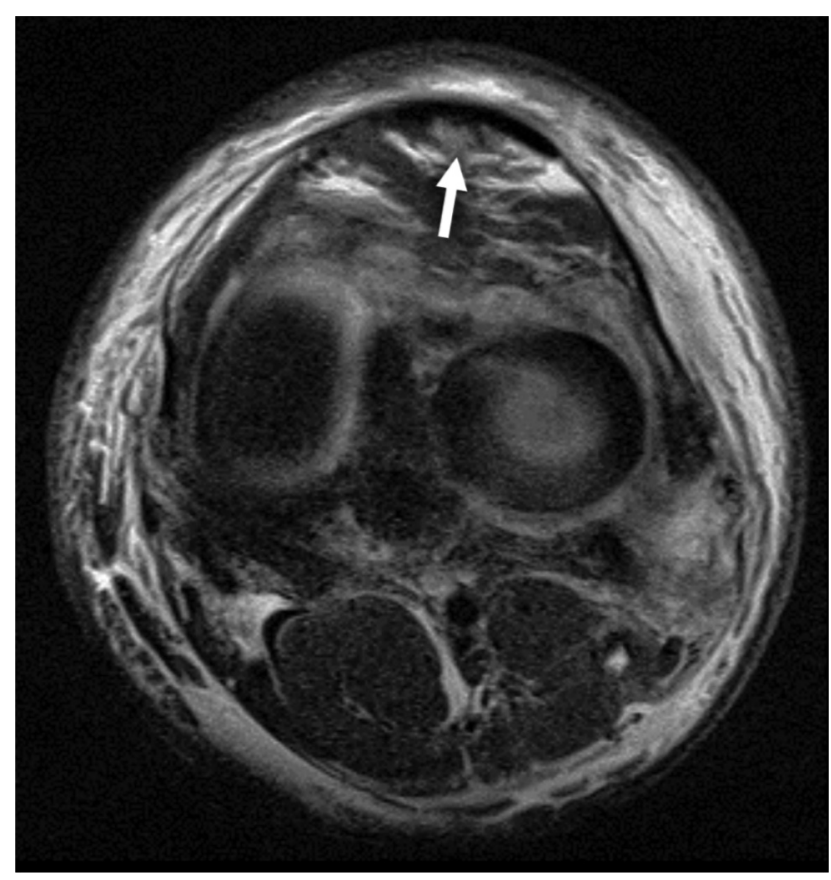

Fig. 5 Axial T2-weithted image demonstrated complete tear of the femoral attachment of the MPFL (arrow). MPFL, medial patellofemoral ligament.

ligament. However, unlike patellar dislocations, there is no evidence of transient patellar dislocation with impaction of the medial patella with the anterior lateral femoral condyle. The proposed mechanism of MPFL tears is stretching and elongation of the ligament from tibiofemoral malalignment ${ }^{13}$ (- Figs. 4 and $\mathbf{5}$ ).

\section{Tendinous Injury}

Patella and quadricep tendon tears are relatively uncommon injuries. Direct trauma may cause an isolated tear of the quadriceps or patellar tendons. Such injury typically will not completely disable the extensor hood, since the medial and lateral retinacula remain intact; therefore, some extensor function will persist. ${ }^{14,15}$

Indirect trauma, on the other hand, can result in complete transection of the extensor mechanism, resulting in complete loss of active or passive extension. Normal tendons usually do not rupture under stress. ${ }^{14}$ In healthy patients, tendons are able to tolerate up to 17.5 times the normal body weight. ${ }^{16}$ Patients with systemic illnesses that result in tendon decline are at higher risk for tendinous injuries. ${ }^{16}$ These include chronic renal failure, rheumatoid arthritis, diabetes, systemic steroid use, hyperparathyroidism, gout, fluoroquinolone use, and connective tissue disorder.

The literature is sparse regarding tendon injuries of the extensor mechanism in the setting of knee dislocations. ${ }^{13,17}$ Tendinous injuries do occur, are less common than MPFL injuries, and are usually partial tendon tears instead of complete ruptures. In one study, approximately one-third of individuals with knee dislocations had evidence of partial tears of the patellar tendon at MRI. ${ }^{13}$ These injuries manifested as diffuse increased signal throughout the course of the tendon - Fig. 6 . The clinical significance of these tendon changes, however, is 


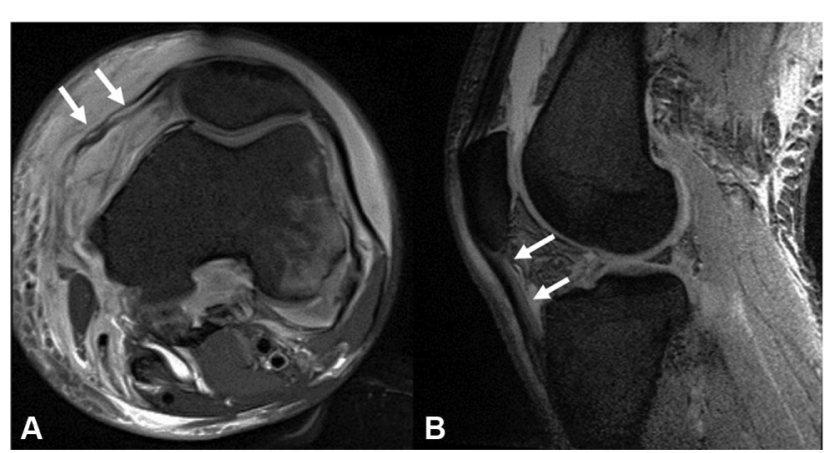

Fig. 6 Axial (A) and sagittal (B) T2-weighted images demonstrate diffuse high signal throughout the patellar tendon (arrows).

unknown and may be well tolerated by individuals. Complete patellar tendon tears requiring surgical repair however, do occur but are uncommon. In one study, complete patellar tendon tears were identified in 2 of 14 patients with knee dislocations. One was as an avulsion of the distal tibial insertion while the other was associated with a comminuted patellar fracture. ${ }^{13}$ Other authors have published similar results of proximal or distal patellar tendon injuries including distal patellar tendon avulsions and patellar fractures. ${ }^{17}$ Neither of these studies reported injuries to the quadriceps tendon and to the authors knowledge, quadriceps tendon injures following knee dislocation have not been reported in the literature. If quadriceps tendon tears occur following knee dislocations, they are likely to be a very rare events.

\section{Conclusion}

Knee dislocations are uncommon but devastating injuries most often affecting the major stabilizers of the knee. Extensor mechanism injuries following knee dislocation include patellar fractures, patellar tendon, and MPFL tears. Tears of the MPFL are common and may result in patellar instability. Physicians should therefore be aware of this potential complication. Most patellar tendon injuries are partial tears; however, complete tendon tears may occur usually at the proximal or distal attachment sites. MRI is considered the gold standard for evaluating these injuries due to its superior contrast resolution.
Conflict of Interest

None declared.

\section{References}

1 Shelbourne KD, Porter DA, Clingman JA, McCarroll JR, Rettig AC. Low-velocity knee dislocation. Orthop Rev 1991;20(11):995-1004

2 O'Malley M, Reardon P, Pareek A, Krych A, Levy BA, Stuart MJ. Extensor mechanism disruption in knee dislocation. J Knee Surg 2016;29(04):293-299

3 Kapur S, Wissman RD, Robertson M, Verma S, Kreeger MC, Oostveen RJ. Acute knee dislocation: review of an elusive entity. Curr Probl Diagn Radiol 2009;38(06):237-250

$4 \mathrm{Yu}$ JS, Goodwin D, Salonen D, et al. Complete dislocation of the knee: spectrum of associated soft-tissue injuries depicted by MR imaging. Am J Roentgenol 1995;164(01):135-139

5 Kennedy JC. Complete dislocation of the knee joint. J Bone Joint Surg Am 1963;45:889-904

6 Goebel CP, Domes C. Classifications in brief: the Schenck classification of knee dislocations. Clin Orthop Relat Res 2020;478(06):1368-1372

7 Helito PVP, Peters B, Helito CP, Van Dyck P. Imaging evaluation of the multiligament injured knee. Ann Joint 2018:3. Doi: 10.21037/ aoj.2018.09.03

8 Yablon CM, Pai D, Dong Q Jacobson JA. Magnetic resonance imaging of the extensor mechanism. Magn Reson Imaging Clin N Am 2014;22(04):601-620

9 Aframian A, Smith TO, Tennent TD, Cobb JP, Hing CB. Origin and insertion of the medial patellofemoral ligament: a systematic review of anatomy. Knee Surg Sports Traumatol Arthrosc 2017;25 (12):3755-3772

10 Galla M, Lobenhoffer P. Patella fractures [in German]. Chirurg 2005;76(10):987-997, quiz 998-999

11 Melvin JS, Mehta S. Patellar fractures in adults. J Am Acad Orthop Surg 2011;19(04):198-207

12 Pengas IP, Assiotis A, Khan W, Spalding T. Adult native knee extensor mechanism ruptures. Injury 2016;47(10):2065-2070

13 Wissman RD, Verma S, Kreeger M, Robertson M. Extensor mechanism injuries in tibiofemoral dislocations. J Comput Assist Tomogr 2009;33(01):145-149

14 Lee D, Stinner D, Mir H. Quadriceps and patellar tendon ruptures. J Knee Surg 2013;26(05):301-308

15 Yu JS, Petersilge C, Sartoris DJ, Pathria MN, Resnick D. MR imaging of injuries of the extensor mechanism of the knee. Radiographics 1994;14(03):541-551

16 Zernicke RF, Garhammer J, Jobe FW. Human patellar-tendon rupture. J Bone Joint Surg Am 1977;59(02):179-183

17 Tzurbakis M, Diamantopoulos A, Xenakis T, Georgoulis A. Surgical treatment of multiple knee ligament injuries in 44 patients: $2-8$ years follow-up results. Knee Surg Sports Traumatol Arthrosc 2006;14(08):739-749 